\title{
Reconstructing WIMP Properties in Direct Detection Experiments Including Galactic Dark Matter Distribution Uncertainties
}

\author{
Louis E. Strigari \\ Kavli Institute for Particle Astrophysics and Cosmology, Stanford University, Stanford, \\ California 94305-4085, USA \\ Roberto Trotta \\ Imperial College London, Astrophysics Group, Blackett Laboratory, Prince Consort Road, \\ London SW7 2AZ, UK
}

\begin{abstract}
We present a new method for determining Weakly Interacting Massive Particle (WIMP) properties in future tonne scale direct detection experiments which accounts for uncertainties in the Milky Way (MW) smooth dark matter distribution. Using synthetic data on the kinematics of MW halo stars matching present samples from the Sloan Digital Sky Survey, complemented by local escape velocity constraints, we demonstrate that the local dark matter density can be constrained to $\sim 20 \%$ accuracy. For low mass WIMPs, we find that a factor of two error in the assumed local dark matter density leads to a severely biased reconstruction of the WIMP spin-independent cross section that is incorrect at the $15 \sigma$ level. We show that this bias may be overcome by marginalizing over parameters that describe the MW potential, and use this formalism to project the accuracy attainable on WIMP properties in future 1 ton Xenon detectors. Our method can be readily applied to different detector technologies and extended to more detailed MW halo models.
\end{abstract}




\section{Introduction}

The detection of dark matter via elastic scatterings in underground detectors is a foremost goal of experimental physics in the coming years. Upper limits on the spin-independent WIMPnucleon cross section [1, 2, 3] are now beginning to carve into the parameter space of the constrained Minimal Supersymmetric Standard Model (cMSSM) [4, 5], which provides a well-motivated theoretical framework for the dark matter. Favored MSSSM parameter space is expected to be fully probed as future detectors reach the tonne scale.

A robust interpretation of the experimental limits (or signals) from detectors requires an understanding of uncertainties associated with the distribution of dark matter in the Milky Way (MW), specifically in the vicinity of the solar neighborhood. Though long regarded a nuisance in the prediction of scattering event rates [6], the potential for the uncertainty in the local dark matter distribution to bias the reconstruction of the WIMP mass and cross section has yet to be fully quantified.

Here we study how well, in a realistic scenario, forthcoming direct detection experiments can do in reconstructing the WIMP mass and cross section, accounting for an uncertain smooth component of the dark matter distribution, which is independently constrained using spectroscopic observations of distant halo stars [7, 8] and measurements of the escape speed near the solar circle [9]. We quantify the bias introduced in the reconstruction of WIMP properties when the uncertainty in the MW halo model is neglected. The results we present are specifically focused on the spin-independent WIMP-nucleon cross section, but are otherwise independent of the specific particle physics framework.

\section{Milky Way Halo}

Rates in direct detection experiments are proportional to the dark matter density at the solar radius. However the potential at the solar radius is likely dominated by baryons, so a selfconsistent approach must model the combined potentials of baryons and dark matter. Disk stars on circular orbits trace the local potential [10], and the dark matter contribution may be determined via continuation of constraints at large radii and under the assumption of a smooth dark matter density profile. This approach is of course subject to systematic uncertainties in the parameterization of the halo potential, though it provides a starting point for understanding the relative contributions from each of the key MW mass components. As we argue here, even a simplified MW model already represents a very considerable improvement in bringing under control systematic errors in reconstructed WIMP properties.

\subsection{Model for Mass Distribution}

We consider a MW halo model that includes a central bulge, disk, and dark halo [9, 11]. The bulge is modeled as a spherically-symmetric Hernquist potential, $\phi_{\text {bulge }}(r)=-G M_{\text {bulge }} /(r+$ $c_{0}$ ), with $c_{0} \sim 0.6 \mathrm{kpc}$ and a total mass $M_{\text {bulge }}=1.5 \times 10^{10} \mathrm{M}_{\odot}$. The disk surface density is taken

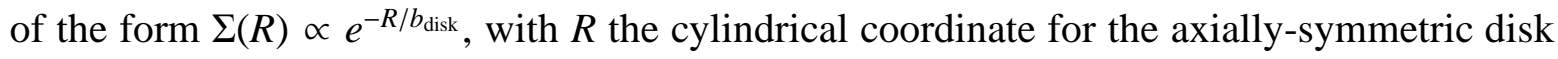
and its scalelength is $b_{\text {disk }} \simeq 4 \mathrm{kpc}$, which is included as a free parameter in our conservative 
model (see below). The potential from this component is clearly non-spherical, though it may be fairly accurately modeled by a spherical distribution that has the same mass interior to a Galactocentric radius $r=R$. The spherically-symmetric disk potential is taken as $\phi_{\text {disk }}=-G M_{\text {disk }}\left(1-e^{-r / b_{\text {disk }}}\right) / r$, where the total disk mass is $M_{\text {disk }}=5 \times 10^{10} \mathrm{M}_{\odot}$. The peak circular velocity for the spherical fit to the disk potential at $\sim 2 b_{\text {disk }}$ is $\sim 15 \%$ less than the circular velocity of the exponential disk and asymptotes to $\lesssim 5 \%$ of the true mass distribution for large radii [12]. For the dark matter halo we take a five parameter model,

$$
\rho(r)=\frac{\rho_{0}}{\left(r / r_{0}\right)^{a}\left[1+\left(r / r_{0}\right)^{b}\right]^{(c-a) / b}} .
$$

The escape velocity, $v_{\text {esc }}$, and the circular velocity at the solar radius, $v_{0}$, are determined from the combined potential of the three components.

As is the case with any parametric model, the results we present will likely vary if the model is not an accurate description of the true Galaxy. As such, we view our analysis as means of estimating uncertainties and bias on key dark matter parameters for a well-defined, though perhaps simplified, MW model, and acknowledge that the above model provides a first step towards consideration of a wider range of Galactic models in this context. For example, to better model observations [13] triaxial models for the MW halo may be considered which increase the dark matter density in the disk. However, on the theoretical side there are uncertainties to the predicted MW halo shape, e.g. simulations suggest that adding gas cooling tends to make halos more spherical [14].

\subsection{Stellar Kinematics}

From the above parametric model for the Galaxy, we simulate stellar kinematics data which we then employ to determine the accuracy with which the particle properties of the dark matter and its astrophysical distribution can be reconstructed. Our synthetic data sample consists of 2000 stars distributed uniformly with Galactocentric radii in the range $5-40 \mathrm{kpc}$, similar to the distribution in recent Sloan Digital Sky Survey measurements [11]. The distance, $d$, from a halo star to the Sun is given by $d^{2}=r^{2}+r_{\odot}^{2}-2 r r_{\odot} \cos \theta$, so that the line-of-sight velocity to the star is $\dot{d}=\dot{r}\left(r / d-r_{\odot} \cos \theta / d\right)+r r_{\odot} \dot{\theta} \sin \theta / d$. Here $\theta$ is the spherical polar angle and $r_{\odot}=8.5 \mathrm{kpc}$ is the distance from the Sun to the Galactic center, which we keep fixed. The line-of-sight velocity dispersion is defined as the average of $\dot{d}^{2}$ over the solid angle in Galactic coordinates, $\sigma_{\text {los }}^{2} \equiv\left\langle\dot{d}^{2}\right\rangle$. Performing this average gives [15],

$$
\sigma_{\text {los }}=\sqrt{1-A(r) \beta(r)} \sigma_{r}
$$

where

$$
A(r)=\frac{r^{2}+r_{\odot}^{2}}{4 r^{2}}-\frac{\left(r^{2}-r_{\odot}^{2}\right)^{2}}{8 r^{3} r_{\odot}} \ln \left|\frac{r+r_{\odot}}{r-r_{\odot}}\right| .
$$

The velocity anisotropy parameter is $\beta(r)=1-\sigma_{\theta}^{2} / \sigma_{r}^{2}$, which we assume to be constant as a function of radius.

Assuming that the dark matter halo and the tracer population of halo stars are in equilibrium, the radial jeans equation for the intrinsic, or statistical, dispersion $\sigma_{r}$ is

$$
\frac{d\left(\rho_{\star} \sigma_{r}^{2}\right)}{d r}+\frac{2 \rho_{\star} \sigma_{r}^{2} \beta(r)}{r}=-G \rho_{\star} \frac{d \phi}{d r}
$$


where $\rho_{\star}(r)$ is the spatial density profile for the tracer population. The potential on the right-hand side is the sum of the potential from the disk, bulge, and the dark matter,

$\phi=\phi_{\text {disk }}+\phi_{\text {bluge }}+\phi_{\text {dm }}$. The density of halo stars is modeled to fall off according to $\rho_{\star} \propto r^{-3.5}$ beyond $10 \mathrm{kpc}$ and remain constant for $r \leq 10 \mathrm{kpc}$ [16].

\section{Direct Detection}

To calculate WIMP-nucleon recoil event rates, we must estimate both the local WIMP density and the velocity distribution of the dark matter. For the latter, we appeal to recent results from high resolution numerical simulations, which find only mild deviations from an isotropic and Maxwellian velocity distribution in the solar neighborhood [17, 18, 19]. This implies 10\% deviations in recoil event rates relative to the standard isotropic Maxwellian assumption [20]. Motivated by these results, we assume an isotropic Maxwellian form for the dark matter velocity distribution, characterized by $v_{\mathrm{esc}}, v_{0}$, and local density $\rho(8.5 \mathrm{kpc})$ [21], which are all computed from our MW model described above and constrained as explained below.

We consider the case of a Xe target, and assume everywhere a 1 tonne-yr exposure. In order to model the energy resolution of the detector, the recoil energy spectrum is smoothed with an energy-dependent Gaussian window function of standard deviation $\sigma_{E}(E)=1.5 \sqrt{E}$. For definiteness, our results below are obtained by considering 5 equally-spaced energy bins within the recoil range $5-30 \mathrm{keV}$, and adopting an independent Poisson likelihood in each bin, with negligible background counts. However, our results are insensitive to the details of the binning scheme and to the precise choice of the upper cutoff in the recoil spectrum. The nuclear form factor for spin-independent scatterings is taken to be the standard Helm form [21]. For the sake of simplicity, we do not include the yearly variation in the event rate resulting from the projection of the Earth's orbital velocity on the Galactic plane; we find that including this effect has negligible effect on the results we present.

\section{Statistical methodology}

We consider the following fiducial parameters for the Milky Way: $\Theta=\left\{\rho_{0}, r_{0}, \beta, a, b, c, b_{\text {disk }}\right\}$ $=\left\{7.4 \times 10^{6} \mathrm{M}_{\odot} \mathrm{kpc}^{-3}, 20 \mathrm{kpc}, 0,1,1,3,4 \mathrm{kpc}\right\}$. This set of dark halo parameters is broadly motivated by numerical simulations of Milky Way mass halos [22], and as discussed above the mass and scalelength of the disk, $b_{\text {disk }}$, are of the order observed for the Milky Way [23]. For this set of parameters the local dark matter density is $0.32 \mathrm{GeV} \mathrm{cm}^{-3}$, the escape velocity $v_{\text {esc }}=547 \mathrm{~km} \mathrm{~s}^{-1}$ and the circular velocity $v_{0}=209 \mathrm{~km} \mathrm{~s}^{-1}$. We find that the results presented below are insensitive to similar sets of fiducial model parameters, provided that they roughly match both the local dark matter density and the mass and circular velocity of the Milky Way. The likelihood is centered around the fiducial values, and is the product of three components: the projected direct detection constraints, the projected stellar kinematics constraints, and present-day escape velocity measurements.

We define the likelihood for the projected velocity component along the line-of-sight to a given star to be Gaussian with zero mean and a variance given by the $\sigma_{\text {los }}$. The total 
likelihood is then obtained by multiplication of the Gaussian likelihood for each star. The dispersion in the likelihood is dominated by the intrinsic dispersion at a given radius, i.e. we ignore contributions to the likelihood that involve uncertainty on the measured velocity of each star. Assuming that the model parameters only enter through the velocity dispersion, it is straightforward to derive the Fisher information matrix, which is defined as the second derivative of the log of the likelihood function, with respect to the model parameters, $\left\langle\partial^{2} \mathcal{L} / \partial \Theta_{a} \partial \Theta_{b}\right\rangle$. For our likelihood the Fisher matrix is simply given by

$$
F_{a b}=\sum_{l=1}^{N} \frac{1}{2} \frac{1}{\sigma_{\mathrm{los}, l}^{4}} \frac{\partial \sigma_{\mathrm{los}, l}^{2}}{\partial \Theta_{a}} \frac{\partial \sigma_{\mathrm{los}, l}^{2}}{\partial \Theta_{b}} .
$$

Written in this form we assume that the mean velocity does not depend on our model parameters; this is likely to be a good assumption if we consider that the intrinsic dispersion of the halo stars dominates the contribution due to ordered rotational motion.

We take the constraint on the escape velocity to be Gaussian with mean $\bar{v}_{\text {esc }}=544 \mathrm{~km}$ $\mathrm{s}^{-1}$ and standard deviation $\sigma=33 \mathrm{~km} \mathrm{~s}^{-1}$ [9]. For simplicity we do not consider additional constraints on the MW mass distribution that come from the inner and outer rotation curves or from the motions of stars in the solar neighborhood, as considered in Refs. [24, 23, 25]. Our motivation for including only the halo stars and the escape velocity constraints in our data set is that these quantities are the most direct tracers of the MW dark matter halo. Given these inputs, the projected constraints on both the local dark matter density and the reconstructed of WIMP properties we present below should be viewed as a conservative estimate of the errors attainable on these parameters.

We adopt a Metropolis-Hastings algorithm to perform a Markov chain Monte Carlo scan over the parameters defining the MW halo model described above, augmented by the WIMP mass $m_{\chi}$ and its spin-independent scattering cross section $\sigma_{p}^{S I}$. We assume flat priors on all MW halo parameters over the ranges $\left\{\log \left[\rho_{0} /\left(\mathrm{M}_{\odot} \mathrm{kpc}^{-3}\right)\right], r_{0} / \mathrm{kpc}, \beta, a, b, c, b_{\text {disk }} / \mathrm{kpc}\right\}=\{[5$ : 8], [10:60], [-1:1], [0:2], [0:2], [2:4], [2:6]\}, as well as flat priors over sufficiently wide ranges of $\log m_{\chi}$ and $\log \sigma_{p}^{S I}$. We find that our results are insensitive to the details of the prior choice.

\section{Results}

We study three representative cases, which differ in the number of halo parameters that are marginalized over. Our fixed model takes $\Theta$ to be fixed to an assumed value, which might not match the correct one. This corresponds to neglecting all astrophysical uncertainties while not including any of the astrophysical constraints. Our baseline model varies the following three parameters: $\left\{\log \rho_{0}, r_{0}, \beta\right\}$, which are constrained using the astrophysical data and marginalized over when considering direct detection constraints, while the remaining four parameters are fixed to their fiducial value. In addition to the three parameters above, our conservative model also marginalizes over the remaining four parameters of the halo model, $\left\{a, b, c, b_{\text {disk }}\right\}$.

Figure 1 shows the 1D posterior marginal probabilities for several parameters for the 

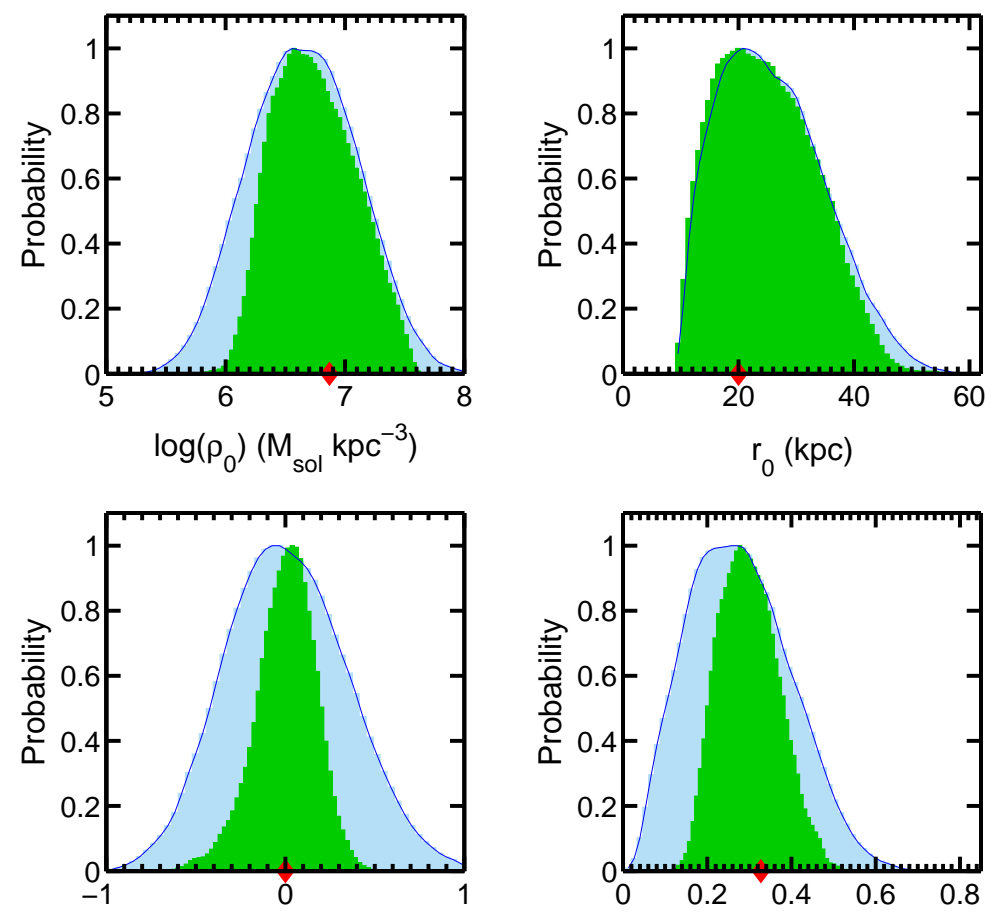

$\beta$

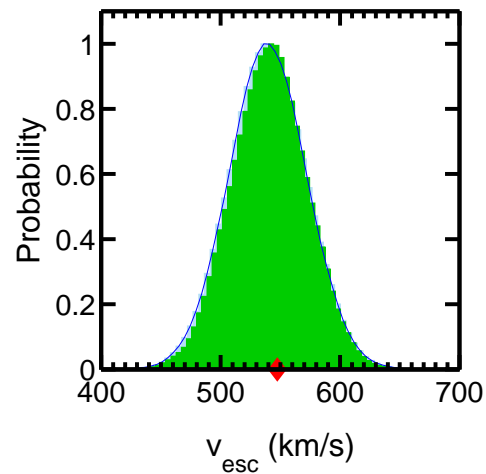

$$
\rho(\mathrm{r}=8.5 \mathrm{kpc})\left(\mathrm{GeV} \mathrm{cm}{ }^{-3}\right)
$$

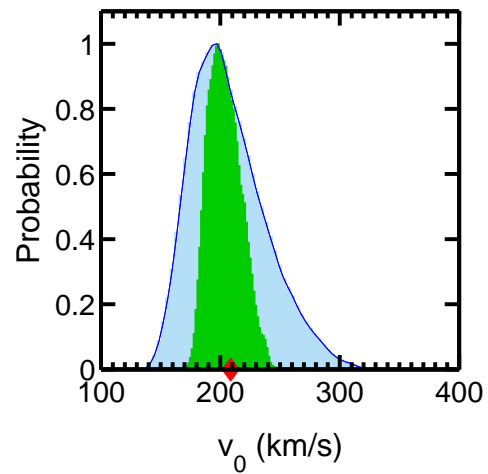

Figure 1. Reconstruction of 3 halo model parameters $\left(\log \rho_{0}, r_{0}, \beta\right)$ and constraints on WIMP scattering rates-related quantities at the solar circle $\left(\rho(8.5 \mathrm{kpc}), v_{\mathrm{esc}}, v_{0}\right)$ using 2000 halo stars and a local determination of the escape velocity. Green (tighter) and blue (wider) curves show the results for the 3 parameter (baseline) and 7 parameter (conservative) halo model. The red diamond is the true value.

baseline and the conservative models, after the halo stars and escape velocity constraints have been applied. The distributions are correctly peaked around the true value and in particular the density of dark matter at $8.5 \mathrm{kpc}$ can be constrained to $22 \%(37 \%)$ at $1 \sigma$ in the baseline (conservative) model. We find that the local escape velocity constraint is important in tightening the bounds on the WIMP local density.

Figure 2 depicts the reconstruction of $m_{\chi}$ and $\sigma_{p}^{S I}$ for a 1 tonne-yr Xe exposure for the baseline (filled/green) and conservative (filled/blue) models, assuming a fiducial value $\sigma_{p}^{S I}=10^{-9} \mathrm{pb}$. We show the case of a low mass $\left(m_{\chi}=50 \mathrm{GeV}\right.$, left panel $)$ and a high 

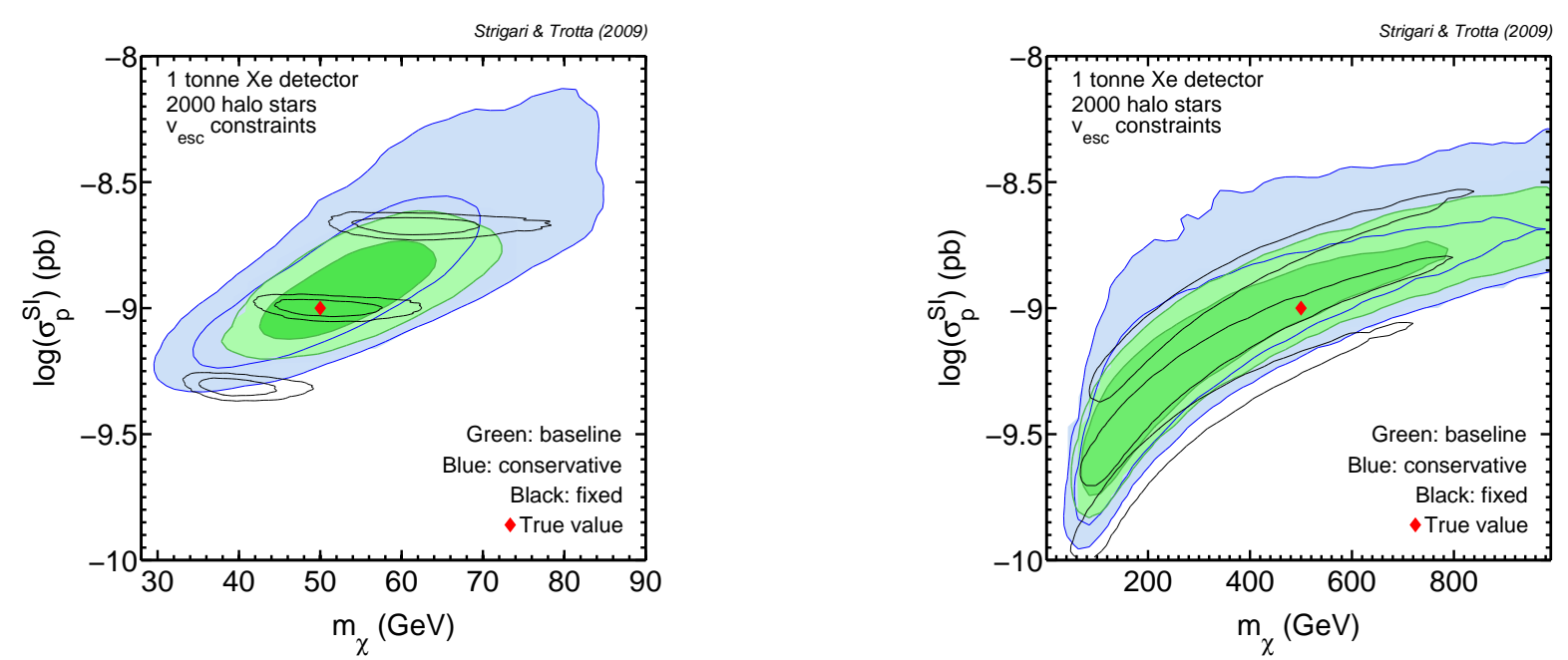

Figure 2. Reconstruction of $m_{\chi}, \sigma_{p}^{S I}$ under various assumptions: dark matter halo parameters fixed to assumed values (solid, black), marginalizing over baseline halo model (filled/green), marginalizing over conservative halo model (filled/blue). In all cases inner and outer contours represent $68 \%$ and $95 \%$ c.l. limits. The red diamond gives the true value. The left panel is for a $50 \mathrm{GeV}$ WIMP mass, the right panel assumes a $500 \mathrm{GeV}$ WIMP (In the right panel, we only show $68 \%$ c.l. for the case of fixed galactic parameters for clarity). The lower (upper) solid black contours illustrate the bias in the reconstruction assuming incorrect values for the local dark matter density a factor of 2 above (below) the true value.

mass WIMP ( $m_{\chi}=500 \mathrm{GeV}$, right panel), to highlight the difference between WIMP mass scales that are well-constrained and poorly constrained [26, 27, 28]. At $m_{\chi}=50 \mathrm{GeV}$, the degeneracy between $m_{\chi}$ and $\sigma_{p}^{S I}$ is broken by recoil energy spectral information; we find that a minimum of 3 evenly spaced bins suffices to break the degeneracy. The fiducial model with $m_{\chi}=50 \mathrm{GeV}\left(m_{\chi}=500 \mathrm{GeV}\right)$ produces 383 (79) events within our energy window for a tonne-yr exposure for Xe.

Figure 2 also shows the bias in the reconstruction resulting from assuming the incorrect local dark matter density. For a $50 \mathrm{GeV}$ WIMP, fixing the local dark matter density a factor of two above or below the true value biases the reconstruction of the WIMP cross section by $\sim 15 \sigma$. The effect is less severe for a $500 \mathrm{GeV}$ WIMP, of order $\sim 5 \sigma$, though this case is intrinsically much less constrained. This systematic bias would not be detectable by the usual goodness-of-fit test, for in all three cases depicted in Figure 2 the reduced chi-square of the best-fit point is statistically indistinguishable. Figure 2 clearly shows the necessity of accounting for uncertain astrophysics in the determination of $m_{\chi}, \sigma_{p}^{S I}$, even in a simplified MW halo model. For the well constrained $50 \mathrm{GeV}$ WIMP case, marginalizing over the halo model parameters correctly recovers the true point with no bias, while the error in the mass increases from $9 \%$ to $14 \%$ (24\%) for the baseline (conservative) model. The impact on the accuracy on $\log \sigma_{p}^{S I}$ is more severe, increasing the relative error by a factor of 5 under the baseline model and by a factor of 20 for the conservative case. However, this reduced statistical accuracy is compensated by a greatly increased robustness to systematic bias. 


\section{Conclusion}

We have presented an analysis of the constraints attainable on WIMP parameters using future direct detection experiments. Our key results are twofold: first we have quantified how well WIMP properties can be constrained for an optimistic particle mass in tonne scale detectors, and second we have demonstrated that including an explicit model of the MW halo can overcome a potentially severe $(\sim 15 \sigma)$ bias in the reconstruction of WIMP parameters when the wrong fiducial values for the halo are assumed. In future analyses our MW halo model can be refined to include triaxial shapes for the dark and luminous components. The contribution from dark matter substructure [29, 18] or a rotating component [30] may be considered. Further one may account for the non-Maxwellian velocity distribution [31], and a multi-component spectral fit to the WIMP and astrophysical background spectra may be incorporated [32, 33]. An analysis along these lines will be crucial to interpret the limits and measurements from forthcoming direct detection experiments.

Acknowledgments- We thank Henrique Araujo, Laura Baudis, Blas Cabrera, Jodi Cooley-Sekula, and Alastair Currie for several important discussions. Support for LES was provided by NASA through Hubble Fellowship grant HF-01225.01 awarded by the Space Telescope Science Institute, which is operated by the Association of Universities for Research in Astronomy, Inc., for NASA, under contract NAS 5-26555. RT would like to thank the EU

FP6 Marie Curie Research and Training Network "UniverseNet" (MRTN-CT-2006-035863) for partial support.

[1] CDMS Collaboration, Z. Ahmed et. al., Search for Weakly Interacting Massive Particles with the First Five-Tower Data from the Cryogenic Dark Matter Search at the Soudan Underground Laboratory, Phys. Rev. Lett. 102 (2009) 011301, arXiv:0802.3530|.

[2] J. Angle et. al., Limits on spin-dependent WIMP-nucleon cross-sections from the XENON10 experiment, Phys. Rev. Lett. 101 (2008) 091301, arXiv:0805.2939].

[3] V. N. Lebedenko et. al., Result from the First Science Run of the ZEPLIN-III Dark Matter Search Experiment, arXiv:0812.1150

[4] L. Roszkowski, R. Ruiz de Austri, and R. Trotta, Implications for the Constrained MSSM from a new prediction for $b \rightarrow$ s $\gamma$, JHEP 07 (2007) 075, [arXiv: 0705.2012].

[5] R. Trotta, F. Feroz, M. P. Hobson, L. Roszkowski, and R. Ruiz de Austri, The Impact of priors and observables on parameter inferences in the Constrained MSSM, JHEP 12 (2008) 024, |arXiv:0809.3792].

[6] G. Jungman, M. Kamionkowski, and K. Griest, Supersymmetric dark matter, Phys. Rept. 267 (1996) 195-373, [hep-ph/9506380].

[7] M. I. Wilkinson and N. W. Evans, The Present and Future Mass of the Milky Way Halo, Mon. Not. Roy. Astron. Soc. 310 (1999) 645, [astro-ph/9906197.

[8] T. Sakamoto, M. Chiba, and T. C. Beers, The Mass of the Milky Way: Limits from a Newly Assembled Set of Halo Objects, Astron. Astrophys. 397 (2003) 899-912, [astro-ph/0210508].

[9] M. C. Smith et. al., The RAVE Survey: Constraining the Local Galactic Escape Speed, Mon. Not. Roy. Astron. Soc. 379 (2007) 755-772, [astro-ph/0611671].

[10] T. Sumi et. al., Probing the Galactic Potential with Next-Generation Observations of Disk Stars, arXiv:0903.4002.

[11] SDSS Collaboration, X. X. Xue et. al., The Milky Way's Circular Velocity Curve to 60 kpc and an Estimate of the Dark Matter Halo Mass from Kinematics of 2400 SDSS Blue Horizontal Branch Stars, Astrophys. J. 684 (2008) 1143-1158, [arXiv:0801.1232].

[12] J. Binney and S. Tremaine, Galactic dynamics. Princeton, NJ, Princeton University Press, 1987, 747 p., 
1987.

[13] M. R. Merrifield, The Galactic Halo and CDM, astro-ph/0310497.

[14] S. Kazantzidis et. al., The Effect of Gas Cooling on the Shapes of Dark Matter Halos, Astrophys. J. 611 (2004) L73-L76, astro-ph/0405189].

[15] W. Dehnen, D. McLaughlin, and J. Sachania, The velocity dispersion and mass profile of the Milky Way, Mon. Not. Roy. Astron. Soc. 369 (2006) 1688-1692, [astro-ph/0603825].

[16] E. F. Bell et. al., The accretion origin of the Milky Way's stellar halo, Astrophys. J. 680 (2008) 295-311, [arXiv:0706.0004].

[17] S. H. Hansen, B. Moore, M. Zemp, and J. Stadel, A universal velocity distribution of relaxed collisionless structures, JCAP 0601 (2006) 014, [astro-ph/0505420].

[18] M. Vogelsberger et. al., Phase-space structure in the local dark matter distribution and its signature in direct detection experiments, Mon. Not. Roy. Astron. Soc. 395 (May, 2009) 797-811, [arXiv:0812.0362].

[19] M. Fairbairn and T. Schwetz, Spin-independent elastic WIMP scattering and the DAMA annual modulation signal, JCAP 0901 (2009) 037, |arXiv:0808.0704].

[20] N. W. Evans and J. H. An, Distribution function of the dark matter, Phys. Rev. D73 (2006) 023524, [astro-ph/0511687.

[21] J. D. Lewin and P. F. Smith, Review of mathematics, numerical factors, and corrections for dark matter experiments based on elastic nuclear recoil, Astropart. Phys. 6 (1996) 87-112.

[22] J. F. Navarro, C. S. Frenk, and S. D. M. White, A Universal Density Profile from Hierarchical Clustering, Astrophys. J. 490 (1997) 493-508, [astro-ph/9611107.

[23] L. M. Widrow, B. Pym, and J. Dubinski, Dynamical Blueprints for Galaxies, Astrophys. J. 679 (June, 2008) 1239-1259, arXiv:0801.3414].

[24] W. Dehnen and J. Binney, Mass models of the Milky Way, Mon. Not. Roy. Astron. Soc. 294 (1998) 429, [astro-ph/9612059].

[25] R. Catena and P. Ullio, A novel determination of the local dark matter density, arXiv:0907.0018

[26] N. Bernal, A. Goudelis, Y. Mambrini, and C. Munoz, Determining the WIMP mass using the complementarity between direct and indirect searches and the ILC, JCAP 0901 (2009) 046, [arXiv:0804.1976].

[27] A. M. Green, Determining the WIMP mass from a single direct detection experiment, a more detailed study, JCAP 0807 (2008) 005, [arXiv: 0805. 1704].

[28] C.-L. Shan, Determining the Mass of Dark Matter Particles with Direct Detection Experiments, arXiv:0903.4320

[29] M. Kamionkowski and S. M. Koushiappas, Galactic Substructure and Direct Detection of Dark Matter, Phys. Rev. D77 (2008) 103509, [arXiv:0801.3269].

[30] J. I. Read et. al., A dark matter disc in the Milky Way, arXiv:0901.2938.

[31] A. H. G. Peter, Dark matter in the solar system I: The distribution function of WIMPs at the Earth from solar capture, arXiv:0902.1344

[32] J. Monroe and P. Fisher, Neutrino Backgrounds to Dark Matter Searches, Phys. Rev. D76 (2007) 033007, arXiv:0706.3019.

[33] L. E. Strigari, Neutrino Coherent Scattering Rates at Direct Dark Matter Detectors, arXiv:0903.3630. 